\title{
Concentrate for Intravesical Solution Dosage Form
}

National Cancer Institute

\section{Source}

National Cancer Institute. Concentrate for Intravesical Solution Dosage Form. NCl

Thesaurus. Code C149392.

Liquid preparation intended to be diluted in the specified liquid to obtain an intravesical solution. 